BMJ Open

Diabetes

Research

\& Care

\title{
Visual impairment and mortality in patients with type 2 diabetes
}

\author{
Volkert Siersma, ${ }^{1}$ Rasmus Køster-Rasmussen, ${ }^{1}$ Christine Bruun, ${ }^{1}$ \\ Niels de Fine Olivarius, ${ }^{1}$ Audun Brunes (i) ${ }^{2}$
}

To cite: Siersma V, Køster-Rasmussen R, Bruun C, et al. Visual impairment and mortality in patients with type 2 diabetes. BMJ Open Diab Res Care 2019;7:e000638. doi:10.1136/ bmjdrc-2018-000638

Received 4 December 2018 Revised 12 September 2019 Accepted 18 September 2019

Check for updates

(C) Author(s) (or their employer(s)) 2019. Re-use permitted under CC BY-NC. No commercial re-use. See rights and permissions. Published by BMJ.

${ }^{1}$ The Research Unit for General Practice and Section of General Practice, Department of Public Health, University of Copenhagen, Copenhagen, Denmark

${ }^{2}$ Section for Trauma, Catastrophes and Forced Migration-Adults and Elderly, Norwegian Centre for Violence and Traumatic Stress Studies (NKVTS), Oslo, Norway

Correspondence to Dr Audun Brunes; audun.brunes@nkvts.no

\section{ABSTRACT}

Objective To evaluate whether visual acuity impairment was an independent predictor of mortality in patients with type 2 diabetes.

Research design and methods This is a 19-year followup of a cohort of 1241 patients newly diagnosed with type 2 diabetes and aged 40 years or over. Visual acuity was assessed by practicing ophthalmologists both at diabetes diagnosis and after 6 years. The logarithmic value of the visual acuity (logMAR) was the exposure. Multivariable Cox regression models were adjusted for multiple potential confounders including cardiovascular disease, and censored for potential mediators, that is, fractures/trauma. Primary outcomes were from national registers: all-cause mortality and diabetes-related mortality.

Results Visual impairment at diabetes diagnosis was robustly associated with subsequent 6 -year all-cause mortality. Per 1 unit reduced logMAR acuity the incidence rate of all-cause mortality increased with $51 \%$ (adjusted HR: $1.51 ; 95 \% \mathrm{Cl} 1.12$ to 2.03 ) and of fractures/trauma with $59 \%$ (HR: $1.59 ; 95 \% \mathrm{Cl} 1.18$ to 2.15 ), but visual acuity was not associated with diabetes-related mortality. After censoring for fractures/trauma, visual acuity was still an independent risk factor for all-cause mortality (HR: 1.68; $95 \% \mathrm{Cl} 1.23$ to 2.30 ). In contrast, visual acuity 6 years after diabetes diagnosis was not associated with the subsequent 13 years' incidence of any of the outcomes, as an apparent association with all-cause mortality and diabetes-related mortality was explained by confounding from comorbidity.

Conclusions Visual acuity measured by ophthalmologists in patients newly diagnosed with type 2 diabetes was an independent predictor of mortality in the short term.

\section{INTRODUCTION}

Visual impairment is a common consequence of type 2 diabetes, affecting about $4 \%-16 \%$ of patients. ${ }^{1-3}$ The global epidemic of type 2 diabetes has led to an increase in the number of microvascular and macrovascular conditions related to vision loss. For example, between 1990 and 2010 the worldwide prevalence of visual impairment caused by diabetic retinopathy increased by $64 \%$ for moderate to severe visual impairment and $27 \%$ for blindness. ${ }^{4}$ Current clinical guidelines recommend annual or biannual screening of patients with diabetes in order to prevent or delay the onset of sight-threatening conditions. ${ }^{5}$

\section{Significance of this study}

What is already known about this subject?

- Visual acuity loss is a common consequence of type 2 diabetes, and may complicate diabetes self-management.

- Using data from a sample of 1381 patients newly diagnosed with type 2 diabetes, this study aimed to assess whether visual acuity loss was an independent risk factor for mortality in this population.

What are the new findings?

- Impaired visual acuity at diagnosis was associated with increased all-cause mortality, independent of other risk factors for mortality.

How might these results change the focus of research or clinical practice?

- Practitioners in primary care should consider screening patients with type 2 diabetes for visual acuity loss soon after diagnosis.

Patients with both diabetes and visual impairment could be at risk of entering a 'vicious cycle' of worsening health. Visual impairment has a huge impact on the performance of self-care activities, including preparing healthy meals, exercising, and taking insulin and medications that help patients to maintain a steady blood glucose level. ${ }^{6}$ In addition, past research has shown that vision impairment may amplify the adverse effects of diabetes, in which patients with both diabetes and visual impairment are at greater risk than sighted patients of experiencing long-term diabetes complications, functional limitations, accidents, mental distress, loneliness, and social disadvantages such as unemployment and poorer access and utilization of healthcare services. ${ }^{6-10}$ Diabetes and its associated complications could also affect people's ability to manage and adjust to their vision loss. ${ }^{6}$

Results from meta-analytic reviews show that the presence of type 2 diabetes doubles the risk of premature mortality from all causes $^{11} 12$ and cardiovascular diseases. ${ }^{11}$ 
While health conditions like hyperglycemia and hypertension have been identified as independent predictors of mortality in patients with type 2 diabetes, ${ }^{13}$ little is known about visual impairment as a possible mortality risk factor of diabetes. ${ }^{14-17}$ Of the published studies on this subject, few have controlled for important mortality risk factors of diabetes, such as diabetes status and chronic conditions. ${ }^{16}$ In addition, the former studies have either sampled patients with visual impairment caused by diabetic retinopathy ${ }^{14-16}$ or blind people with early-onset type 1 diabetes, ${ }^{17}$ typically patient groups with long diabetes durations. It is well known that diabetes duration predicts both diabetes complications ${ }^{18}$ and excess risk of death. ${ }^{13}$ Hence, to understand the complex interplay between visual impairment and type 2 diabetes on morbidity and mortality, there is a need for studies on patient groups being early in the course of the disease.

Visual acuity can easily be accessed in the clinic and it would be of general clinical interest to uncover whether this information carries any prognostic information in itself. The objective of the present study was to evaluate whether visual acuity impairment was an independent risk factor for mortality in patients with type 2 diabetes, and thereby to clarify whether such relation was explained by confounding, notably from cardiovascular disease, or by mediation through concurrently increased incidence of fractures/trauma.

\section{METHODS}

\section{Patients}

For the Diabetes Care in General Practice study (DCGP), ${ }^{19}$ 474 primary care physicians in Denmark volunteered to recruit all of their patients who were aged 40 years or older and diagnosed with diabetes during the 3-year

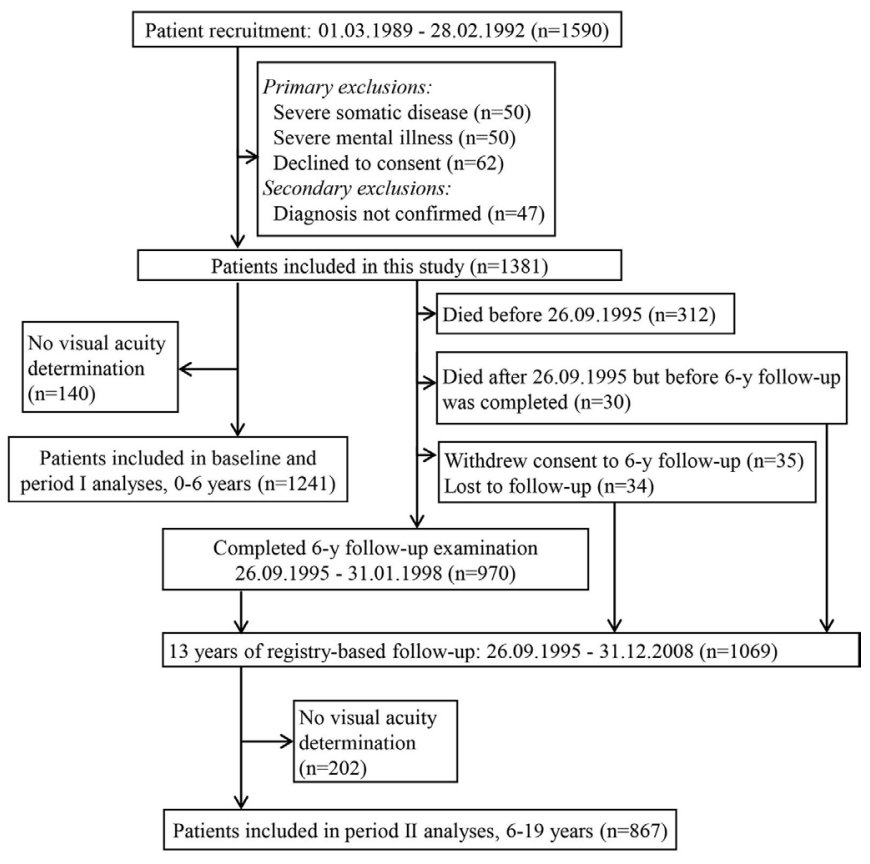

Figure 1 Patient flow through study. inclusion period in 1989-1992 (figure 1). Following recruitment, the diabetes diagnosis was confirmed by a single fasting whole blood/plasma glucose concentration $(\geq 7.0 / 8.0 \mathrm{mmol} / \mathrm{L})$ measured at a major laboratory. The protocol-based exclusion criteria were life-threatening somatic disease, severe psychiatric disease, or unwillingness to participate. Previous to the inclusion period, the physicians were randomized to give either personal, structured care or routine care to the included patients until a 6-year follow-up which started on 26 September 1995.

\section{Assessments and definitions}

After the diabetes diagnosis and in connection with the 6-year follow-up examination, the patients were referred to a practicing ophthalmologist who did a standard eye examination and funduscopy. Visual acuity (the eye's ability to resolve fine details) and visual field are the two visual functions assessed in the classification of visual impairment. ${ }^{20}$ Visual acuity with the best correction for each eye was estimated with an optotype chart, usually a Snellen chart, at $6 \mathrm{~m}$ (20 feet). Visual acuity was recorded as decimal digits which, according to usual clinical and administrative practice in Denmark, categorizes the patients, according to the visual acuity in the best seeing eye, into normal vision $(\geq 0.5)$, moderate to severe visual impairment $(<0.5$ and $>0.1)$ or blind $(\leq 0.1)$. For adequate analysis, this visual acuity was converted to $\log$ MAR: minus one times the logarithm of visual acuity of the best seeing eye ${ }^{21}$; increments of $1 \log$ MAR unit from 0 to 3 are approximately equivalent to decimal visual acuity values of $1.00,0.35,0.15$, and 0.05 , respectively. The result of the funduscopy was recorded as one of six response categories $^{1}$ : no diabetic lesions, microaneurysms only, background retinopathy without or with maculopathy, and proliferative retinopathy without or with new vessels on the optic disc. Concurrently, information about other retinal pathologies such as eye operations, glaucoma, presence of cataract and reasons for impaired vision was obtained. In $69 \%$ of the patients the same ophthalmologist did the eye examination at diagnosis and at 6 -year follow-up.

Primary outcomes were all-cause mortality and diabetes-related mortality and a secondary outcome was the incidence of fractures/trauma, each over the period from diagnosis to the 6-year follow-up and over the period from the 6-year follow-up until 31 December 2008. Vital status and emigration status of the participants were obtained from The Danish Civil Registration System. ${ }^{22}$ Causes of death were taken from The Danish Register of Causes of Death. ${ }^{23}$ Incidence of somatic disease was taken from The Danish Register of Causes of Death and The Danish National Patient Register, which includes all inpatient and outpatient hospital contacts, but no primary care contacts. ${ }^{23-25}$ Diabetes-related deaths were defined as fatal myocardial infarction, stroke, renal disease, hyperglycemia, hypoglycemia, peripheral vascular disease and sudden death. ${ }^{26}$ Fractures/trauma was defined as 
all diagnoses beginning with 8, 90-98, 991, 992, or 996 (International Classification of Diseases Eighth Revision, ICD-8), or all S-diagnoses and diagnoses beginning with T0-T2, T30-T35, T51-T59, T61-T65, T79, or T90-T98 (ICD-10).

In questionnaires filled in at diabetes diagnosis and 6-year follow-up patients gave information about familiar disposition to diabetes, education, residence, physical activity, smoking habits, and whether they lived alone. The general practitioners recorded clinical information on the patients including body height and weight, blood pressure, heart rate, and history of hypoglycemic events, cardiovascular disease, peripheral neuropathy, amputations and cancer. A more detailed description of the variables and definitions has previously been published. ${ }^{26}$ Cardiovascular disease was defined as history of myocardial infarction and/or history of stroke and/or angina pectoris and/or intermittent claudication and/or absent arterial pulses on both feet and/or amputation on the lower extremities; peripheral neuropathy was defined as lack of a sense of pinprick and/or touch of cotton wool on at least one foot and/or absent patellar reflex on at least one knee; and cancer was defined as self-report of any cancer except benign skin cancers.

\section{Assays}

Fasting blood samples and urine samples were collected at diabetes diagnosis and 6-year follow-up. Measurements of HbA1c (reference range $36-57 \mathrm{mmol} / \mathrm{mol}(5.4 \%-$ $7.4 \%$ ); using the method created by the Diabetes Control and Complications Trial (DCCT), the interval may be translated into $29-50 \mathrm{mmol} / \mathrm{mol}(4.8 \%-6.7 \%))$, total cholesterol, triglycerides, serum creatinine and urinary albumin were centralized. ${ }^{1}$

\section{Statistical analyses}

The increase in incidence of the outcomes attributable to visual impairment was assessed with hazard ratios (HRs) for a unit increase in logMAR acuity estimated from a multivariable Cox regression model on time to death/ event. Two sets of analyses were performed: (1) one for outcomes in the period from diabetes diagnosis to year 6 (period I), and (2) one for outcomes in the period from year 6 to year 19 after the diabetes diagnosis (period II). Visual acuity at diagnosis was the exposure for period I, and visual acuity at year 6 was the exposure for period II. Risk times were censored at the end of the follow-up period or emigration. The analysis was split in these two periods in order to investigate whether visual impairment had a different role at diagnosis than at a later stage of the disease.

The multivariable analyses were adjusted for groups of possible confounders which were (A) background variables: sex, age; (B) sociodemographics: familial disposition to diabetes, living alone, education, residence; $(\mathrm{C})$ biochemical risk factors: (diagnostic) plasma glucose (for period I), HbAlc (for period II), total cholesterol, fasting triglycerides, urinary albumin, serum creatinine;
(D) clinical risk factors: height (in interaction with sex), weight (in interaction with sex), hypertension, resting heart rate; (E) lifestyle variables: smoking, physical activity, trial arm; (F) chronic conditions: peripheral neuropathy, cardiovascular disease, cancer (former or present); $(G)$ eye pathologies: age-related macular degeneration (AMD), cataract, diabetic retinopathy, other retinopathy, eye pressure.

The combination of the groups of confounders presented in the final multivariable model was determined by sequential backward elimination of the groups for which the $\mathrm{p}$ value of the corresponding likelihood ratio test was higher than 0.05 , until all remaining tests had $p<0.05$. For each regression model the validity of the proportional hazard assumption was assessed by including an interaction with $\log$ (time) and each covariate in the model; a joint test for these interactions tested the assumption. In none of the models the proportional hazard assumption was violated. Patients with missing values were omitted from the analysis. A $p$ value $<0.05$ indicated statistical significance. The statistical analyses were performed by the use of SAS V.9.3.

\section{RESULTS}

Of the 1381 included patients, 1241 and 867 had visual acuity measured at diagnosis and at 6-year follow-up examination, respectively. A small number started insulin treatment within 180 days of diagnosis, so $97.6 \%$ of the patients were considered to have type 2 diabetes. Of the patients assessed at the time of the diabetes diagnosis the prevalence of moderate to severe visual impairment and blindness was $5.4 \%$ (95\% CI 4.1 to 6.7 ) and $0.9 \%$ (95\% CI 0.4 to 1.4), respectively. Among the patients alive and with a valid determination of visual acuity 6 years after the diagnosis, the prevalence of moderate to severe visual impairment and blindness was $6.7 \%$ (95\% CI 5.0 to 8.4) and $2.4 \%$ (95\% CI 1.3 to 3.4 ), respectively. The median time between the first and the second eye examination was 5.6 years (IQR: 5.0-6.3).

At diabetes diagnosis and compared with patients with normal vision, patients with moderate to severe visual impairment or blindness were on average older, more often female, and living alone (table 1). They also had a higher prevalence of hypertension, peripheral neuropathy and cardiovascular disease and they were less physically active. The same pattern was present at diabetes diagnosis and 6 years later. The most commonly diagnosed eye pathologies were AMD and cataract at both diagnosis $(12.4 \%$ and $27.5 \%$, respectively) and follow-up (11.7\% and $33.9 \%$, respectively). Diabetic retinopathy was seen in $4.1 \%$ at diagnosis, and in $13.7 \% 6$ years later.

All-cause mortality according to visual acuity is presented in Kaplan-Meier plots in figure 2. Figure 2A displays a dose-response relationship between visual acuity at diagnosis and all-cause mortality, whereas the death rates were equally high in patients who were blind 


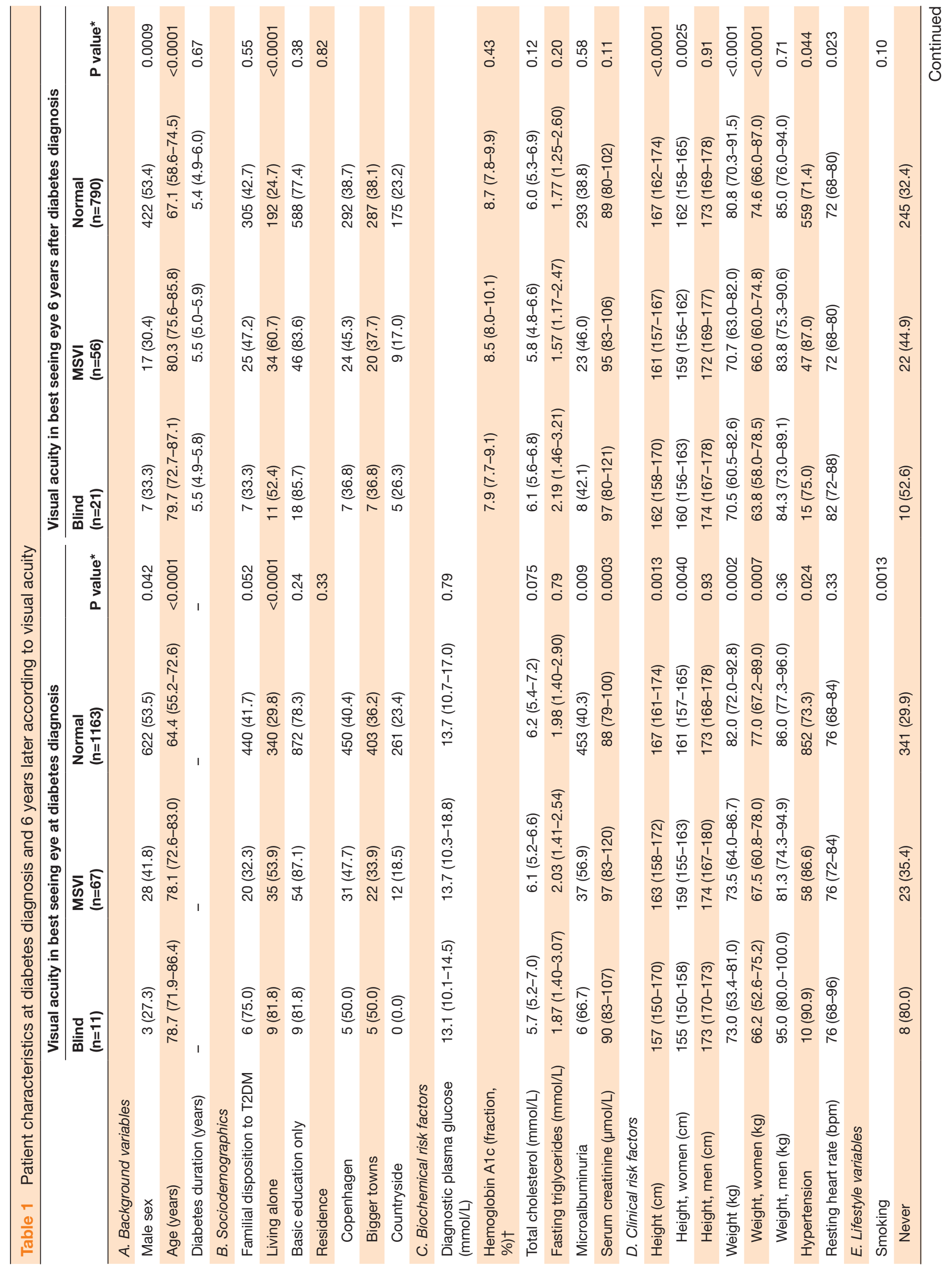




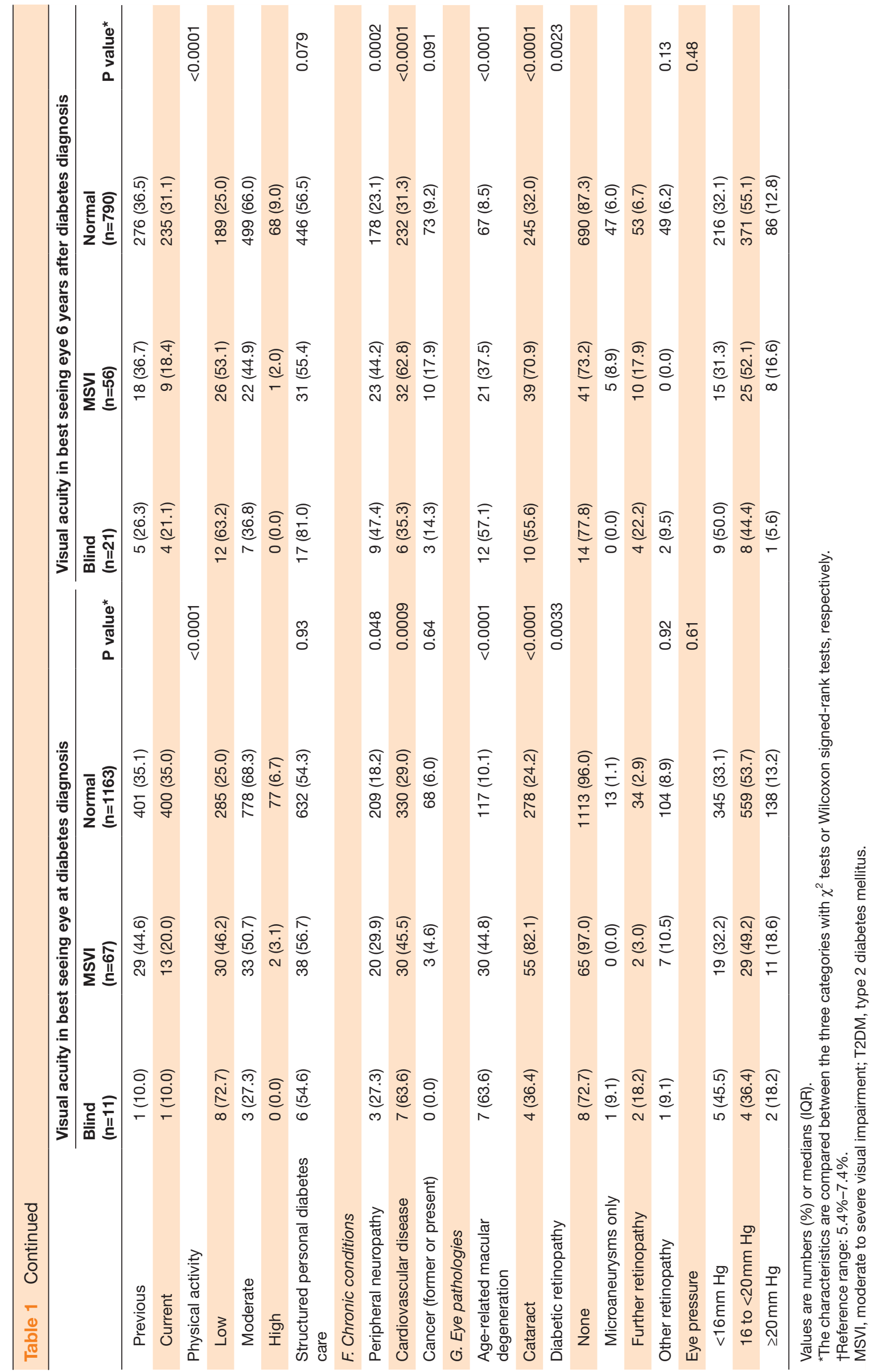



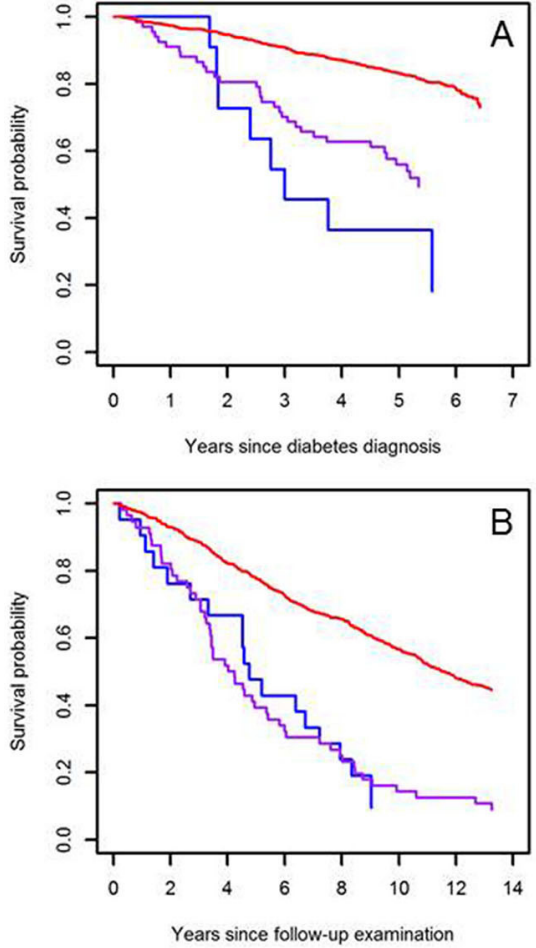

Figure 2 Kaplan-Meier plot of all-cause mortality from diabetes diagnosis $(A)$ and from follow-up after 6 years $(B)$ in categories of visual acuity: normal vision (red), impaired vision (blue), and blind (purple).

or had moderate to severe visual impairment at the 6-year examination (figure 2B).

Table 2 shows that reduced visual acuity ( 1 unit increase in $\log$ MAR) at the baseline examination was significantly associated with all-cause mortality after adjustments for age and sex, after adjustments for various groups of possible confounding factors, as well as after intensive confounder control. However, we did not find any significant associations for diabetes-related mortality in the adjusted models. At the 6-year examination, reduced visual acuity was only associated with all-cause and diabetes-related mortality in some models. The observed unadjusted association was mainly explained by confounding from biochemical risk factors $(\mathrm{C})$, lifestyle variables and trial arm (type of diabetes care) (E), and disease severity $(\mathrm{F})$.

The results on the secondary outcome fractures/ trauma are shown in table 3. Also here reduced visual acuity at diagnosis was seen associated with an increased incidence of fractures/trauma, but visual acuity at the 6-year examination was not. A supplementary analysis of all-cause death where patients were censored for fractures/trauma (in order to compare mortality for those without trauma and thereby effectively correct for the higher mortality in patients who experience trauma) found that visual acuity was still an independent risk factor for all-cause mortality even when patients experiencing a trauma were removed from the analysis (HR: $1.68 ; 95 \%$ CI 1.23 to 2.30$)$.

\section{CONCLUSIONS}

The current study showed that poor visual acuity was associated with increased risk of all-cause mortality in patients newly diagnosed with type 2 diabetes, and the association remained similar or slightly weaker after controlling for possible confounding factors. Moreover, patients with visual impairment at diabetes diagnosis had more accidents but the increased number of fractures and trauma did not explain the excess mortality. At the 6-year examination, visual acuity did not predict mortality or accidents. From a clinical point of view, our results indicate that poor visual acuity could be of particular importance in the clinical decision-making shortly after diabetes diagnosis (period I), while being of less relevance in decisions at a later stage of the disease (period II).

Our findings of higher all-cause mortality risk with reduced baseline levels of visual acuity are consistent with previous studies. ${ }^{14-17}$ For example, Rajala $e t a l^{14}$ found an HR of 5.1 (95\% CI 2.6 to 11.0) for all-cause mortality in patients with visual impairment caused by diabetic retinopathy compared with a group of age and gender-matched adults without diabetes, while sighted patients with diabetes who had been treated previously for retinopathy had an HR of 2.4 (95\% CI 1.1 to 5.6). Our study extends the evidence by demonstrating a relationship between visual impairment and all-cause mortality in a sample of patients with newly diagnosed type 2 diabetes, and hence reducing the possibility of confounding from long-term diabetes complications such as diabetic retinopathy.

In the present study, we observed that patients with both diabetes and visual impairment were more likely than sighted patients to have fractures/trauma, poor lifestyle behaviors, hypertension, neuropathy, and cardiovascular diseases at the baseline examination. Nonetheless, our data do not support a mediating role of these factors in the relation between reduced visual acuity and all-cause mortality. This is in line with the results of a study of 3711 patients with type 2 diabetes, in which diabetes complications accounted for only $10 \%-18 \%$ of the higher mortality risk of impaired visual acuity. ${ }^{16}$ Given the advanced age of our study population and the high proportion of people with age-related eye diseases and cardiovascular conditions at baseline, the higher mortality risk of patients with reduced visual acuity at the baseline assessment may be related to biological ageing. ${ }^{27}$ Indeed, age-related eye diseases, such as AMD and cataract, are considered markers of biological ageing $^{28}$ and might accelerate ageing. ${ }^{27}$ Reduced visual acuity could also reflect ageing changes in the structures of the eyes, as the eye changes shape, the lens loses elasticity, and the number of retinal ganglion cells decreases with advanced age. ${ }^{29}$

The weak adjusted associations of visual impairment assessed 6 years after diabetes diagnosis (period II) with all-cause and diabetes-related mortality were unexpected because of the observed increase in prevalence in visual impairment and diabetic retinopathy during the study period. In the multivariable model the apparent associations were mainly explained by (E) lifestyle variables 
Table 2 The predictive value of visual acuity at diabetes diagnosis and 6 years later for the two primary outcomes: all-cause death and diabetes-related death

\begin{tabular}{|c|c|c|c|c|c|c|}
\hline & \multicolumn{3}{|c|}{$\begin{array}{l}\text { Period I (6 years) } \\
\text { From diabetes diagnosis to } 6 \text { years later }\end{array}$} & \multicolumn{3}{|c|}{$\begin{array}{l}\text { Period II ( } 13 \text { years) } \\
\text { From } 6 \text { to } 19 \text { years after diagnosis }\end{array}$} \\
\hline & $\mathrm{n}$ & HR $(95 \% \mathrm{Cl})$ & $P$ value & $\mathbf{n}$ & HR $(95 \% \mathrm{Cl})$ & $P$ value \\
\hline \multicolumn{7}{|c|}{ All-cause death } \\
\hline Unadjusted & 1241 & 1.64 (1.47 to 1.84$)$ & $<0.0001$ & 867 & 1.53 (1.42 to 1.65$)$ & $<0.0001$ \\
\hline \multicolumn{7}{|l|}{ Adjusted for: } \\
\hline A & 1241 & 1.31 (1.11 to 1.55 ) & 0.0019 & 867 & 1.16 (1.04 to 1.28$)$ & 0.0053 \\
\hline$A+B$ & 1051 & 1.24 (1.02 to 1.51$)$ & 0.032 & 734 & 1.14 (1.02 to 1.28$)$ & 0.025 \\
\hline$A+C$ & 1177 & 1.63 (1.23 to 2.15$)$ & 0.0006 & 746 & 1.11 (0.99 to 1.24$)$ & 0.073 \\
\hline$A+D$ & 1235 & 1.28 (1.07 to 1.53$)$ & 0.0074 & 840 & 1.14 (1.02 to 1.27$)$ & 0.018 \\
\hline$A+E$ & 1213 & 1.27 (1.05 to 1.53$)$ & 0.015 & 818 & $1.08(0.97$ to 1.21$)$ & 0.16 \\
\hline$A+F$ & 1198 & 1.26 (1.07 to 1.50$)$ & 0.0071 & 783 & 1.09 (0.96 to 1.24$)$ & 0.20 \\
\hline$A+G$ & 1101 & 1.32 (1.04 to 1.68$)$ & 0.025 & 718 & 1.28 (1.10 to 1.47$)$ & 0.0006 \\
\hline $\mathrm{A}+\mathrm{C}+\mathrm{E}+\mathrm{F}^{*}$ & 1134 & 1.51 (1.12 to 2.03 ) & 0.0074 & 678 & 1.03 (0.89 to 1.20$)$ & 0.70 \\
\hline \multicolumn{7}{|c|}{ Diabetes-related death } \\
\hline Unadjusted & 1241 & 1.61 (1.39 to 1.87$)$ & $<0.0001$ & 863 & 1.59 (1.46 to 1.74$)$ & $<0.0001$ \\
\hline \multicolumn{7}{|l|}{ Adjusted for: } \\
\hline A & 1241 & 1.20 (0.93 to 1.54$)$ & 0.16 & 863 & 1.23 (1.10 to 1.37$)$ & 0.0004 \\
\hline$A+B$ & 1051 & $1.10(0.82$ to 1.48$)$ & 0.52 & 731 & 1.22 (1.07 to 1.38$)$ & 0.0021 \\
\hline$A+C$ & 1177 & 1.36 (0.94 to 1.97$)$ & 0.11 & 743 & 1.17 (1.03 to 1.33$)$ & 0.018 \\
\hline$A+D$ & 1235 & 1.15 (0.88 to 1.51$)$ & 0.29 & 836 & 1.23 (1.09 to 1.39$)$ & 0.0007 \\
\hline$A+E$ & 1213 & 1.12 (0.83 to 1.50$)$ & 0.46 & 815 & $1.14(1.00$ to 1.29$)$ & 0.046 \\
\hline$A+F$ & 1198 & $1.13(0.87$ to 1.47$)$ & 0.35 & 782 & 1.12 (0.96 to 1.31$)$ & 0.14 \\
\hline$A+G$ & 1101 & $1.17(0.86$ to 1.59$)$ & 0.32 & 714 & 1.33 (1.13 to 1.56$)$ & 0.0004 \\
\hline $\mathrm{A}+\mathrm{C}+\mathrm{E}+\mathrm{F}^{*}$ & 1134 & $1.16(0.77$ to 1.75$)$ & 0.49 & 681 & 1.05 (0.89 to 1.25$)$ & 0.55 \\
\hline
\end{tabular}

Values are numbers and HRs for a 1 unit increase in logMAR (the logarithmic value of visual acuity of the best seeing eye) from multivariable Cox regression models on time to death/event. Models were adjusted for groups of possible confounders: (A) background variables: sex and age; (B) sociodemographics: familial disposition to diabetes mellitus, living alone, education, and residence; (C) biochemical risk factors: diagnostic fasting plasma glucose (for period I), hba1c (for period II), total cholesterol, fasting triglycerides, urinary albumin, and serum creatinine; (D) clinical risk factors: height (in interaction with sex), weight (in interaction with sex), hypertension, and resting heart rate; (E) lifestyle variables: smoking, physical activity and trial arm; (F) chronic conditions: peripheral neuropathy, cardiovascular disease, and cancer (former or present); (G) eye pathologies: age-related macular degeneration (AMD), cataract, diabetic retinopathy, other retinopathy, and eye pressure.

*The combination of the groups of confounders presented in the final multivariable model was determined by sequential backward elimination of the groups for which the $p$ value of the corresponding likelihood ratio test was higher than 0.05 , until all remaining tests had $\mathrm{p}<0.05$. Adjustments after backward elimination turned out to be the same for period I and period II for diabetes-related death, but the adjustment for all-cause death in period II was $A+C+D+E+F$.

(smoking, physical activity and trial arm) and (F) chronic conditions (peripheral neuropathy, cardiovascular disease, and cancer). An interpretation of our findings is that the disease severity leading to cardiovascular morbidity and diabetic retinopathy could have been the main cause of visual impairment in the individuals with highest mortality in the following years after diagnosis. Even at diabetes diagnosis the patients with visual impairment had a significantly higher prevalence of hypertension and cardiovascular disease indicating higher mortality risk. Another possible interpretation is that the diabetes intervention (E) reduced mortality particularly for individuals with visual impairment in period II. ${ }^{30}$ The aim of the intervention with structured personal care was exactly to optimize follow-up and self-care and improve long-term glycemic control reducing morbidity and mortality risks. Lastly, because a high number of participants died during the follow-up period, we cannot rule out the possibility of a healthy worker survivor bias. ${ }^{31}$

\section{Strengths and limitations}

Compared with earlier studies it is a strength that the multivariable analyses were adjusted for disease severity, and that the results were robust after censoring for fractures/ trauma. However, and in resemblance with all observational studies, we cannot rule out the possibility of residual confounding. One factor of concern is related to socioeconomic status. ${ }^{79}$ Although we adjusted for education, living 
Table 3 The predictive value of visual acuity at diabetes diagnosis and 6 years later for the secondary outcome: fractures/ trauma

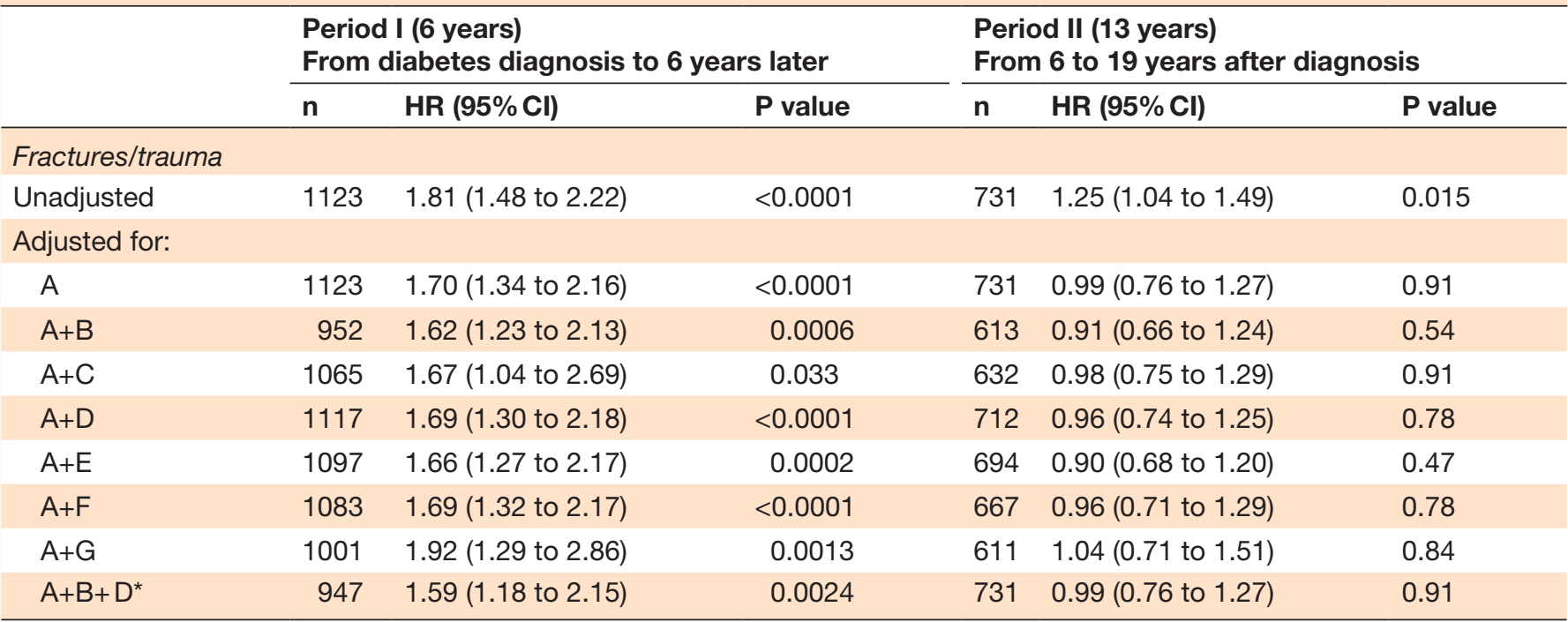

Values are numbers and HRs for a 1 unit increase in logMAR (the logarithmic value of visual acuity of the best seeing eye) from multivariable Cox regression models on time to death/event. Models were adjusted for groups of possible confounders: (A) background variables: sex and age; (B) sociodemographics: familial disposition to diabetes mellitus, living alone, education, and residence; (C) biochemical risk factors: diagnostic fasting plasma glucose (for period I), hba1c (for period II), total cholesterol, fasting triglycerides, urinary albumin, and serum creatinine; (D) clinical risk factors: height (in interaction with sex), weight (in interaction with sex), hypertension, and resting heart rate; (E) lifestyle variables: smoking, physical activity and trial arm; (F) chronic conditions: peripheral neuropathy, cardiovascular disease, and cancer (former or present); (G) eye pathologies: age-related macular degeneration (AMD), cataract, diabetic retinopathy, other retinopathy, and eye pressure.

${ }^{*}$ The combination of the groups of confounders presented in the final multivariable model was determined by sequential backward elimination of the groups for which the $p$ value of the corresponding likelihood ratio test was higher than 0.05 , until all remaining tests had $\mathrm{p}<0.05$. Adjustments after backward elimination turned out to be the same for period I and period II.

arrangements and residence, these are only basic socioeconomic indicators, and we did not have access to data on, for example, occupational status, income and wealth. We expect that the observed associations would have been slightly weaker if we had included a more detailed measure of socioeconomic status in our analyses.

The study population was a representative sample of patients with diabetes in the background population. Yet, the results can only be generalized to adult patients with clinical type 2 diabetes in a western world setting as the etiology of visual acuity loss might differ between high and low-income countries. ${ }^{28}$

Visual acuity was measured twice by an ophthalmologist. Our results were based on best corrected visual acuity at the better seeing eye, while other studies have used presenting, binocular or self-reported visual acuity. Unlike logMAR charts, the Snellen chart, however, has a large-scale increment resulting in a relatively high measurement error. Another factor contributing to measurement variability was differing routines for taking account of visual field loss. The lower precision of the exposure variable, that is, visual acuity, will however only contribute to weaken the strength of the association between vision impairment and mortality.

The involvement of almost all Danish ophthalmologists in the study increased the inter-rater variability, and $10 \%-40 \%$ of sight-threatening eye disease may have been overlooked, ${ }^{32}$ although it can be assumed that the detection rate for eye disease was higher in patients with low visual acuity. Still, in $69 \%$ of the patients the two eye examinations were carried out by the same ophthalmologist. Nonetheless, this measurement error may have reduced our ability to adjust for confounding of the relation between visual acuity and mortality, as the presence of eye diseases is associated with both visual loss and increased mortality.

In conclusion, visual impairment at diabetes diagnosis was an independent risk factor for all-cause mortality during the following 6 years. This association was not explained by cardiovascular disease, disease severity, or fractures/trauma. The visual acuity of a patient with diabetes is an easily accessible clinical measure, also for the primary care practitioner. In order to identify and intensify the treatment of high-risk patients in the daily clinical work, visual acuity can thus be regarded as a risk factor in line with other clinical tests like microalbuminuria, blood pressure and plasma glucose.

Acknowledgements The authors thank the patients, general practitioners and ophthalmologists who participated in the study.

Contributors VS, AB and NdFO developed the research question and wrote the protocol for this follow-up study. NdFO was responsible for the original study design and data collection. NdF0 and VS were responsible for data collection from the registries. VS performed the statistical analyses. All authors made substantial contributions to the analysis and interpretation of data. The paper was written by VS, AB, RKR and CB, and revised by NdFO critically for important intellectual content. NdFO obtained funding and is the guarantor of the study. All authors have approved the final version of the manuscript. 
Funding This work was supported by grants from The Danish Medical Research Council, The Danish Research Foundation for General Practice, The Danish Ministry of Health, The Health Insurance Foundation, The Pharmacy Foundation and the Novo Nordisk Farmaka Denmark.

Competing interests NdFO reports grants from The Danish Medical Research Council, grants from The Danish Research Foundation for General Practice, grants from The Danish Ministry of Health, grants from The Health Insurance Foundation, grants from The Pharmacy Foundation and grants from the Novo Nordisk Farmaka Denmark, during the conduct of the study.

Patient consent for publication Not required.

Ethics approval The approval of the study by the Copenhagen and Frederiksberg Research Ethics Committee (V.100.869/87) included that oral informed consent was mandatory for all patients.

Provenance and peer review Not commissioned; externally peer reviewed.

Data availability statement Data are available upon reasonable request.

Author note Part of the study data are from a clinical trial of diabetes patients (the DCGP Study) while another part is provided by a third party. SAMBA is a unit at Copenhagen University that stores and shares data from epidemiological research in general practice. Anonymized data from the DCGP study can be provided through application to SAMBA by presenting a methodologically sound study protocol and statistical analysis plan. Interested researchers can contact the SAMBA coordination group at tjs657@sund.ku.dk with request for DCGP study data.

Open access This is an open access article distributed in accordance with the Creative Commons Attribution Non Commercial (CC BY-NC 4.0) license, which permits others to distribute, remix, adapt, build upon this work non-commercially, and license their derivative works on different terms, provided the original work is properly cited, appropriate credit is given, any changes made indicated, and the use is non-commercial. See: http://creativecommons.org/licenses/by-nc/4.0/.

ORCID iD

Audun Brunes http://orcid.org/0000-0002-0806-5963

\section{REFERENCES}

1 de Fine Olivarius N, Siersma V, Almind GJ, et al. Prevalence and progression of visual impairment in patients newly diagnosed with clinical type 2 diabetes: a 6 -year follow up study. BMC Public Health 2011;11:80.

2 Miki E, Lu M, Lee ET, et al. The incidence of visual impairment and its determinants in the who multinational study of vascular disease in diabetes. Diabetologia 2001;44 Suppl 2:S31-6.

3 Klein R, Lee KE, Knudtson MD, et al. Changes in visual impairment prevalence by period of diagnosis of diabetes: the Wisconsin epidemiologic study of diabetic retinopathy. Ophthalmology 2009;116:1937-42.

4 Leasher JL, Bourne RRA, Flaxman SR, et al. Global estimates on the number of people blind or visually impaired by diabetic retinopathy: a meta-analysis from 1990 to 2010. Diabetes Care 2016;39:1643-9.

5 American Optometric Association. AOA optometric clinical practice guidelines [article online]. Available: https://www.aoa.org/ optometrists/tools-and-resources/clinical-care-publications/clinicalpractice-guidelines

6 Senra H, Barbosa F, Ferreira P, et al. Psychologic adjustment to irreversible vision loss in adults: a systematic review. Ophthalmology 2015;122:851-61.

7 Cupples ME, Hart PM, Johnston A, et al. Improving healthcare access for people with visual impairment and blindness. BMJ 2012;344:e542.

8 Brunes A, Hansen MB, Heir T. Post-traumatic stress reactions among individuals with visual impairments: a systematic review. Disabil Rehabil 2019;41:2111-8.

9 Crews JE, Jones GC, Kim JH. Double jeopardy: the effects of comorbid conditions among older people with vision loss. J Vis Impair Blind 2006;100(1 Suppl):824-48.
10 Fenwick E, Rees G, Pesudovs K, et al. Social and emotional impact of diabetic retinopathy: a review. Clin Exp Ophthalmol 2012;40:27-38

11 Nwaneri C, Cooper H, Bowen-Jones D. Mortality in type 2 diabetes mellitus: magnitude of the evidence from a systematic review and meta-analysis. Br J Diabetes Vasc Dis 2013;13:192-207.

12 Wright AK, Kontopantelis E, Emsley R, et al. Life expectancy and cause-specific mortality in type 2 diabetes: a population-based cohort study quantifying relationships in ethnic subgroups. Diabetes Care 2017:40:338-45.

13 Engelmann J, Manuwald U, Rubach C, et al. Determinants of mortality in patients with type 2 diabetes: a review. Rev Endocr Metab Disord 2016;17:129-37.

14 Rajala U, Pajunpää H, Koskela P, et al. High cardiovascular disease mortality in subjects with visual impairment caused by diabetic retinopathy. Diabetes Care 2000;23:957-61.

15 Laatikainen L, Ojamo M, Rudanko S-L, et al. Improving visual prognosis of the diabetic patients during the past 30 years based on the data of the Finnish register of visual impairment. Acta Ophthalmol 2016;94:226-31.

16 Cusick M, Meleth AD, Agrón E, et al. Associations of mortality and diabetes complications in patients with type 1 and type 2 diabetes: early treatment diabetic retinopathy study report No. 27. Diabetes Care 2005;28:617-25

17 Grauslund J, Green A, Sjølie AK. Blindness in a 25-year follow-up of a population-based cohort of Danish type 1 diabetic patients. Ophthalmology 2009;116:2170-4.

18 Donnelly R, Emslie-Smith AM, Gardner ID, et al. Vascular complications of diabetes. BMJ 2000;320:1062.

19 Olivarius NF, Beck-Nielsen H, Andreasen AH, et al. Randomised controlled trial of structured personal care of type 2 diabetes mellitus. BMJ 2001;323:970-5.

20 World Health Organization. ICD-10 Version:2016 [article online]. World Health Organization, 2016. Available: http://apps.who.int/ classifications/icd10/browse/2016/en/

21 Holladay JT. Proper method for calculating average visual acuity. $J$ Refract Surg 1997;13:388-91.

22 Pedersen CB. The Danish civil registration system. Scand J Public Health 2011;39(7 Suppl):22-5.

23 Helweg-Larsen K. The Danish register of causes of death. Scand J Public Health 2011;39(7 Suppl):26-9.

24 Lynge E, Sandegaard JL, Rebolj M. The Danish national patient register. Scand J Public Health 2011;39(7 Suppl):30-3

25 Mors O, Perto GP, Mortensen PB. The Danish psychiatric central research register. Scand J Public Health 2011;39(7 Suppl):54-7.

26 Hansen LJ, Siersma V, Beck-Nielsen H, et al. Structured persona care of type 2 diabetes: a 19 year follow-up of the study diabetes care in general practice (DCGP). Diabetologia 2013;56:1243-53.

27 Wang A-G. Visual impairment and mortality: are they related? J Chin Med Assoc 2015;78:137-8.

28 Flaxman SR, Bourne RRA, Resnikoff S, et al. Global causes of blindness and distance vision impairment 1990-2020: a systematic review and meta-analysis. Lancet Glob Health 2017;5:e1221-34.

29 Gittings NS, Fozard JL. Age related changes in visual acuity. Exp Gerontol 1986;21:423-33.

30 Larsen JR, Siersma VD, Davidsen AS, et al. The excess mortality of patients with diabetes and concurrent psychiatric illness is markedly reduced by structured personal diabetes care: a 19-year follow up of the randomized controlled study diabetes care in general practice (DCGP). Gen Hosp Psychiatry 2016;38:42-52.

31 Robins J. A new approach to causal inference in mortality studies with a sustained exposure period-application to control of the healthy worker survivor effect. Mathematical Modelling 1986;7:1393-512.

32 Harding SP, Broadbent DM, Neoh C, et al. Sensitivity and specificity of photography and direct ophthalmoscopy in screening for sight threatening eye disease: the Liverpool diabetic eye study. BMJ 1995;311:1131-5. 